\title{
Nocardiosis in pets: first reported cases in Russia
}

\author{
Roman S. Ovchinnikov*1, Alla G. Gaynullina ${ }^{1}$ \\ ${ }^{1}$ Federal Research Center «All-Russian Research Institute of Experimental Veterenary Medicine (VIEV) n.a. K.I. \\ Skryabin and Y.R. Kovalenko» \\ Laboratory of mycology and antibiotics n.a. A. Sarkisov \\ 109052 Russian Federation, Moscow Ryazansky prospect, 24-1 \\ *Corresponding author. E-mail rsovchinnikov@viev.ru \\ ORCID: https://orcid.org/0000-0001-6634-9879
}

\begin{abstract}
Two cases of cutaneous nocardiosis in a cat and in a dog have been described. Diagnosis was made on basis of direct microscopy and cultural mycological analysis. Phenotypic characteristics of two indigenous Nocardia strains are presented. The strain isolated from cat was presumably identified as $N$. asteroids. There have been no reports of suchlike cases in pets in Russia so far.
\end{abstract}

Keywords: animal nocardiosis; Nocardia; veterinary mycology; actinomycetes; Russian Federation; pet; dermatitis;

\section{Introduction}

Nocardia spp. are soil saprophytes which are able to cause superficial and deep infections in several domestic animals. Nocardiosis is detected in companion animals (dogs and cats), horses and ruminants [1]. Nocardiosis is frequently initiated by inhalation of organism from soil or by skin inoculation through a puncture wounds. Diagnosis is based on the presence of microorganisms in tissue smears as well as on cultural detection of Nocardia spp. Materials may be cultured on chocolate agar, blood agar and Sabouraud agar under aerobic conditions [2]. At least twelve species within the genus Nocardia have been defined by the use of expanded and susceptibility tests and the application of molecular techniques [1].

Susceptibility of animal species can vary from country to country. Bovine mastitis is the most common clinical manifestation of nocardiosis among domestic ruminants in Brazil [3]. In companion animals nocardiosis manifests as cutaneous and subcutaneous pyogranulomatous suppurative lesions and pneumonia. R. Malik [2] reported cutaneous/subcutaneous nocardiosis is the most common clinical form in companion animals in Australia and it is seen almost exclusively in cat. Here we describe two cases of dermatoses in companion animals (cat and dog) caused by Nocardia spp. To the best of our knowledge no cases of animal nocardiosis were reported in Russia until now.

\section{Case description}


Case 1. A 4-year-old Siberian short-hair cat, female, was referred to the Moscow laboratory of veterinary mycology for the mycological examination of skin lesion resembling dermatophytosis. The first clinical signs were detected by the owner at day 21 heretofore the presentation. No topical or systemic treatment was performed.

Case 2. A 7-year old Shi-tsu breed dog, male, was referred to the Moscowlaboratory of veterinary mycology for the mycological examination of skin lesion suspected for fungal infection. The first clinical signs were detected by the owner at 14 days before the presentation. No topical or systemic treatment was performed.

No anamnestic data revealing immunosuppression was recorded in both cases. The physical and mycological examinations were conducted. The examination in Wood's lamp light was performed to detect fluorescence in affected areas. The microscopic examinations of skin scrapings and hairs in $10 \%$ potassium hydroxide wet mount were carried out in order to detect fungal elements (spores and/or mycelium). The direct microscopy was carried out on Microoptix MX-100 microscope (Austria) at x100 and x400 dry magnification. The same clinical material was taken to perform cultural mycological investigation. The material was inoculated on malt-extract agar slants (MEA, Oxoid Ltd, England) and MEA with selective supplements Chloramphenicol and Dermasel (Oxoid Ltd, England). The plates were incubated at $28^{\circ} \mathrm{C}$ up to $21 \mathrm{~d}$ under aerobic conditions.

In case 1 (cat) dermatological examination revealed alopecia, skin scales, crusts, pus discharge on left cheek area. In case $2(\mathrm{dog})$ purulent skin lesion localized near right eye was revealed. No fluorescence in Wood's lamp light was detected in both cases.

The direct microscopic examination of skin samples revealed thin hyaline branching filaments which did not correspond to the fungal hyphae but were more relevant for actinomycetes (Fig. 1). The similar filaments were detected in both animals. No other microorganisms or parasites were revealed. 


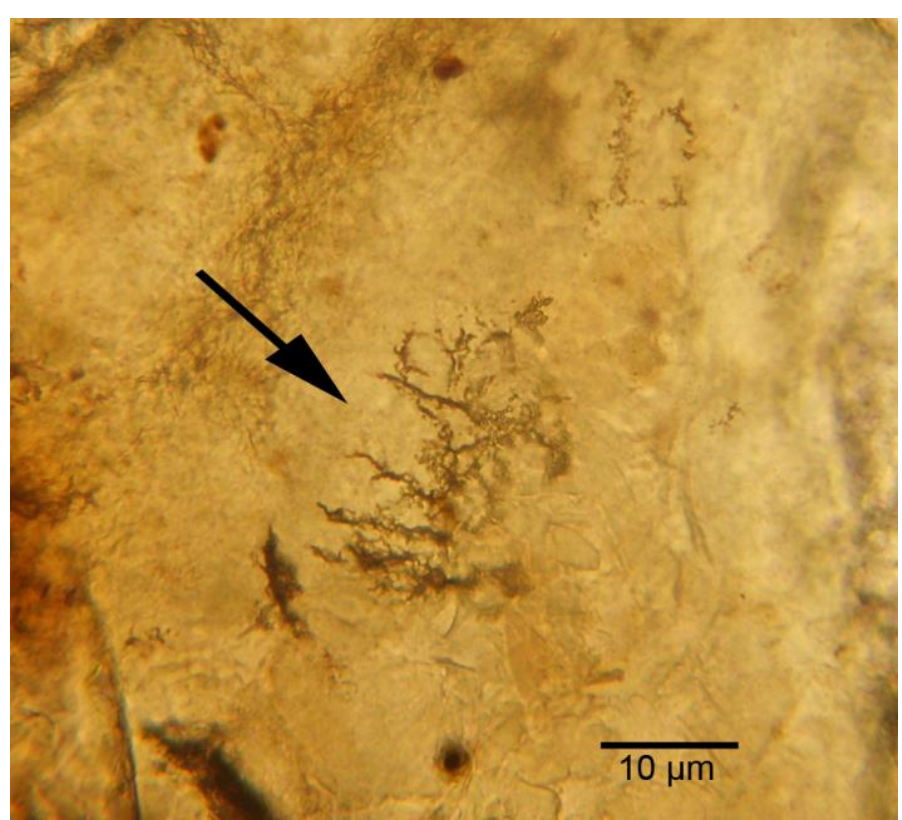

Fig. 1. Appearance of Nocardia spp. in skin scraping. Branching hyaline filaments (unstained wet mount in $10 \%$ potassium hydroxide, optical magnification x 400)

The presence of colonies with very restricted growth was observed on MEA after $7 \mathrm{~d}$. The morphological features were detectable only after 14 days of cultivation. No growth on MEA with selective supplements was detected.

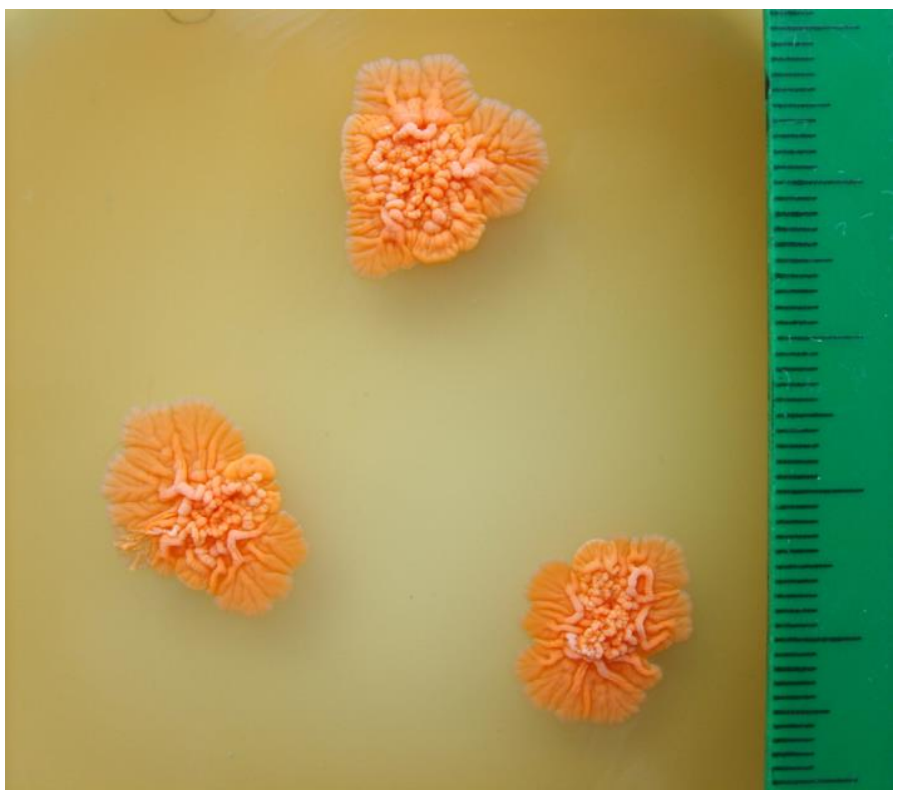

Fig. 2. Colony morphology of Case 1 Nocardia spp. Red-orange wrinkled powdery colony $($ MEA, 14 d) $($ scale bar = $1 \mathrm{~mm}$ )

In case 1 (cat) colonies were red-orange, irregular, convex, with powdery surface, extremely wrinkled, $0,5-1,0 \mathrm{~cm}$ diam. (Fig. 2). On microscopy (unstained wet mount) cocobacillary cells were observed (Fig. 3). 


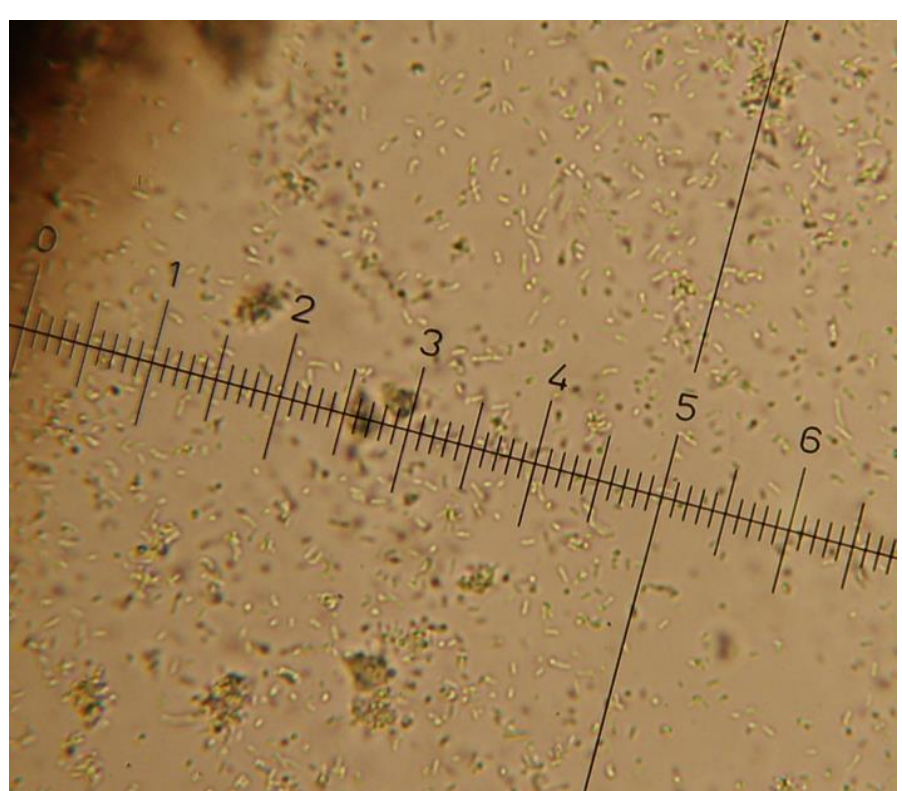

Fig. 3. Microscopy of Case 1 Nocardia spp. culture. Coccobacillary cells (unstained wet mount. Scale bar $=2,5 \mu \mathrm{m}$ )

In case 2 (dog) colonies were colorless, irregular, convex, glistening, extremely wrinkled, 0,5-1,0 cm diam. (Fig. 4).

On microscopy branching hyaline filaments and coccoid cells were observed (Fig. 5).

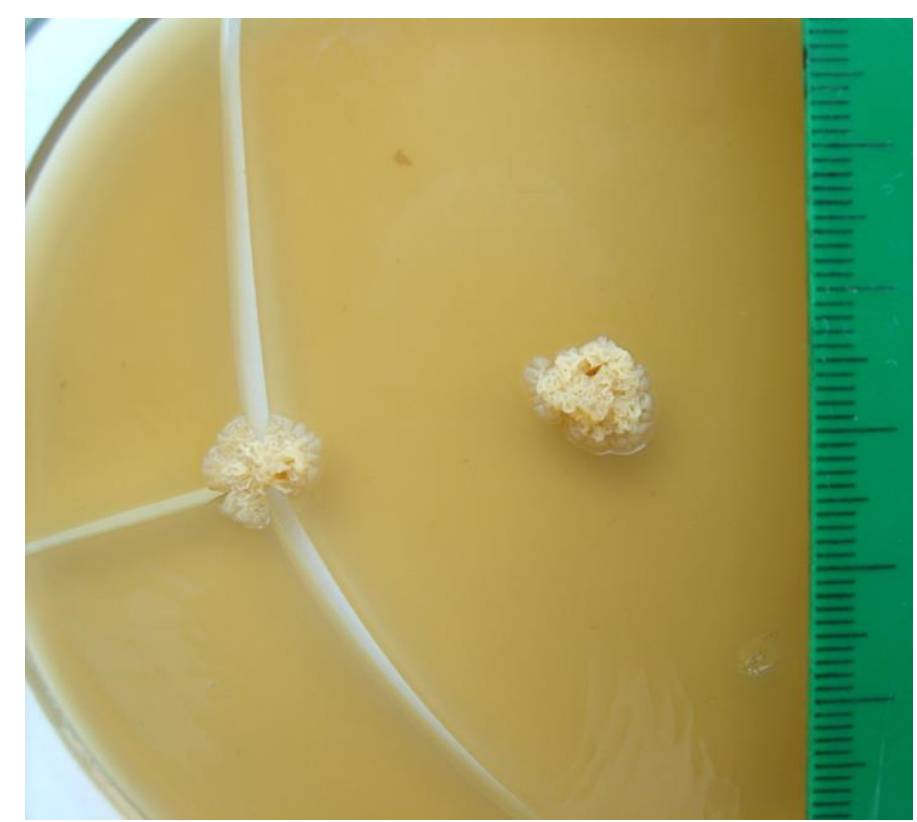

Fig. 4. Colony morphology of Case 2 Nocardia spp. Colorless glistening wrinkled colony $(\mathrm{MEA}, 14 \mathrm{~d})(\mathrm{scale}$ bar = $1 \mathrm{~mm})$ 


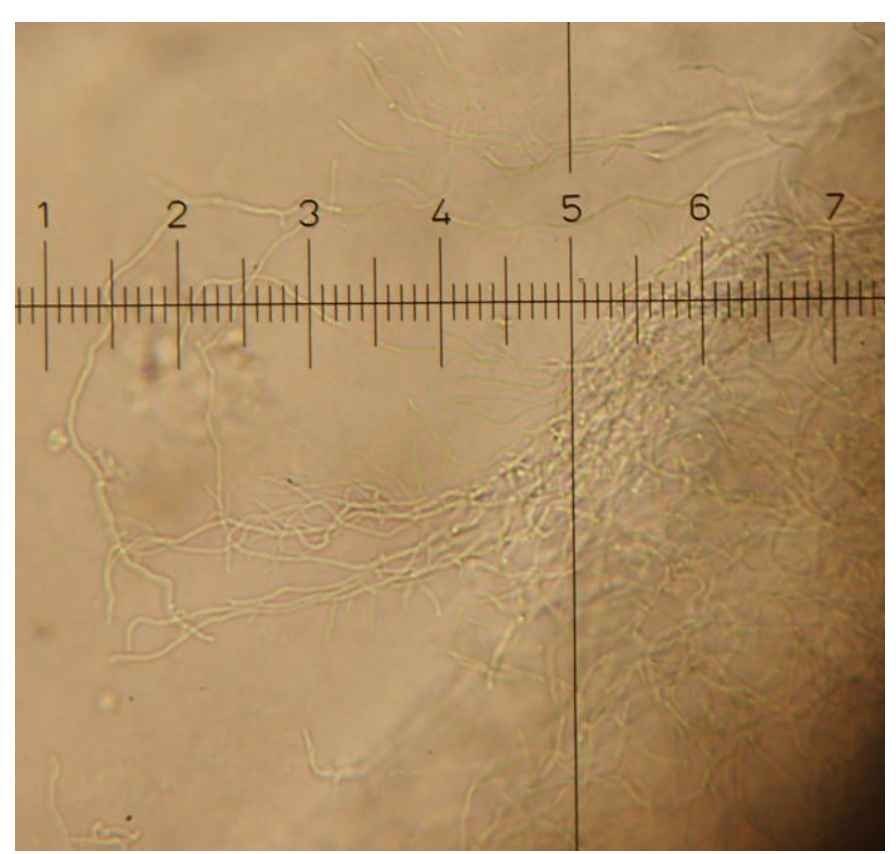

Fig. 5. Microscopy of Case 2 Nocardia spp. culture. Branching filaments (unstained wet mount. Scale bar $=2,5 \mu \mathrm{m}$ )

The cultured microorganisms were catalase and urease positive. Basing on all these findings both microorganisms were identified as Nocardia spp. In case 1 (cat) the colony morphological features were characteristic for Nocardia asteroides, but further molecular studies are needed to confirm this suggestion. Unfortunately, both animals were lost for further follow up.

\section{Discussion}

Nocardial infections (nocardiosis) in companion animals are described as cutaneous and subcutaneous lesions (abscess, mycetoma), often with draining sinuses. Superficial lesions are often associated with invasion of adjacent structures, leading to pulmonary and (or) disseminated diseases. In our case 1 (cat) the lesion was extremely similar to dermatophytosis. Such a similarity can lead to misdiagnose of the disease if proper mycological analysis was not performed.

The nocardiosis in dogs and cats was described in both immunocompetent or immunosuppressed animals. Immunosuppressive infectious diseases in companion animals (distemper in dogs, leukemia and immunodeficiency in cats) are usually described to be co-infected with Nocardia spp. [2], but no such underlying diseases were recorded in our cases. 
In most studies the blood agar and incubation at $37^{\circ} \mathrm{C}$ were used for culturing Nocardia spp., although mycological Sabouraud agar was successfully used too [4]. In such conditions the growth can be detected in 3-7 days. In our study the growth rate was extremely low, that could be connected with temperature mode $\left(28^{0} \mathrm{C}\right.$ in our study instead of $37^{\circ} \mathrm{C}$ in the others). However, our cultural conditions allow to detect dermatophytes and other clinical fungi together with aerobic actinomycetes. Such conditions are mostly applicable in routine mycological diagnostics.

Although nocardiosis has been considered as an emerging disease among domestic animals worldwide, there have been no reports of such cases in Russia so far. The reports of nocardiosis emergency are mainly refer to countries of warm climate such as Australia [2, 5], USA [6], Brazil [1]. However, recently canine nocardiosis was reported in Poland [4]. Thus we can ascertain the spreading of nocardiosis towards the northern areas of Europe.

\section{Conflict of interest statement}

The authors declared no potential conflicts of interest with respect to the research, authorship, and/or publication of this article.

\section{Ethical approval}

This work involved the use of non-experimental animals only (owned pets), and followed established internationally recognized high standards ('best practice') of individual veterinary clinical patient care. Ethical approval from a committee was not necessarily required.

\section{Informed consent}

Informed consent (either verbal of written) was obtained from the owners of all animal(s) described in this work for the procedure(s) undertaken. No animals or humans are identifiable within this publication, and therefore additional informed consent for publication was not required

\section{References}

[1] Malik R. Nocardia infections of the subcutis and skin of cats. In: Fifth world Congress of veterinary dermatology. Clinical program proceedings. Vienna: WCVD 5; 2004, pp. 375-382.

[2] Maraki S, Scoulica E, Alpantaki K, Dialynas M, Tselentis Y. Limphocutaneous nocardiosis due to Nocardia brasiliensis. Diagnost Microbiol Inf Dis 2003; 47:341-344.

[3] Ribeiro M, Salerno T, Mattos-Guaraldi A, Camello T, Langoni H, Siqueira A. Nocardiosis: an overview and additional report of 28 cases in cattle and dogs. Rev Ins. Med Trop S. Paulo 2008; 50(3):177-185 
[4] Golynski M, Szczepanik M, Pomorska D, Wilkolek P. Cutaneous nocardiosis in a dog - clinical case presentation. Bull Vet Inst Pulawy 2006; 50:47-50.

[5] Sivacolundhu RK, O’Hara AJ, Read RA. Thoracic actinomycosis (arcanobacteriosis) or nocardiosis causing thoracic pyogranuloma formation in three dogs. Aust Vet J 2001; 79:398-402.

[6] Kirpensteijn J, Fingland R Cutaneous actinomycosis and nocardiosis in dogs: 48 cases (1980-1990). J Am Vet Med Assoc 1992; 5:917-920. 